\title{
«The brain is not a mental muscle»
}

Dominik Heima,

Rita Schaumann-von Stosch ${ }^{b}$

a Schweizerische Gesellschaft für Traumatologie und

Versicherungsmedizin SGTV

b SUVA, Präsidentin SGTV
Korrespondenz: PD Dr. med. Dominik Heim Chefarzt Chirurgie Spital Frutigen CH-3714 Frutigen

dominik.heim[at]spitalfmi.ch
Das war einer dieser prägnanten Sätze, die einem nach dem Symposium «Sportverletzungen» vom 28. November in der FIFA-Halle in Zürich geblieben sind. Organisiert wurde dieser Anlass von der SUVA, der Schweizerischen Gesellschaft für Traumatologie und Versicherungsmedizin, SGTV, und der Rehaklinik Bellikon. Geladen waren sportinteressierte Traumatologen, Neurologen und Rechtsanwender. Und enttäuscht wurden sie nicht - es war ein hochspannender Anlass, ein sehr sinnvoller medizinischer Winterbeginn, ein Einstimmen auf die Wintersporttraumatologie mit einem Abstecher in den Fussball (auch den American Football), in Kampfsportarten und in die Leichtathletik.

Und Sepp Blatter erinnerte mit seiner kurzen Begrüssungsrede daran, dass dieser sportmedizinische Anlass an einem sehr speziellen, oder eben sehr sinnigen Ort stattfand: der Zentrale des Weltfussballs «Fussball für alle, alle für den Fussball oder - FIFA. For the game. For the world». Dass gerade an diesem Tag über den Arbeitsunfall in einem brasilianischen Fussballstadion in den Medien berichtet wurde, zeigte die Relevanz, die dem Sport in der heutigen Welt zukommt. «Panem et circenses» hiess es schon bei den Römern. Brot haben zwar immer noch nicht alle, Spiele aber gibt es von Sotschi bis Katar - es hat sich in den vergangenen Jahrtausenden in dieser Hinsicht also wenig verändert - der Drang nach Wettbewerb, nach sportlichem Kräftemessen und nach persönlicher Bestleistung ist geblieben. Schade, dass man dabei immer wieder vergisst, dass in der Antike an Olympischen Spielen auch alle Kriege ruhten!

25\% aller Unfälle in der Schweiz sind Sportunfälle, am häufigsten sind sie im Fussball und dann im Skifahren, und 90\% dieser Unfälle betreffen Amateure (Vogt). Das unterstreicht die Bedeutung der Unfall-Prävention, wie sie auch von der Beratungsstelle für Unfallverhütung bfu betrieben wird (Brügger), auch wenn dabei Aufwand und Ertrag nicht ganz einfach und genau abzuschätzen sind (am ehesten noch in den unfallbedingten Arbeitsabwesenheiten). Messen lässt sich dies auch im Profisport in der unfallbedingten Sportabwesenheit. Durch präventive Massnahmen ereignen sich heute, zum Beispiel im Fussball 30\% weniger Unfälle (Dvorak). So führt ein Ellbogencheck an den Kopf automatisch zur roten Karte und damit zum Spielerausschluss, denn «concussion», die Benommenheit nach einem Schlag an den Kopf, bedingt als sich einfach zu mer-

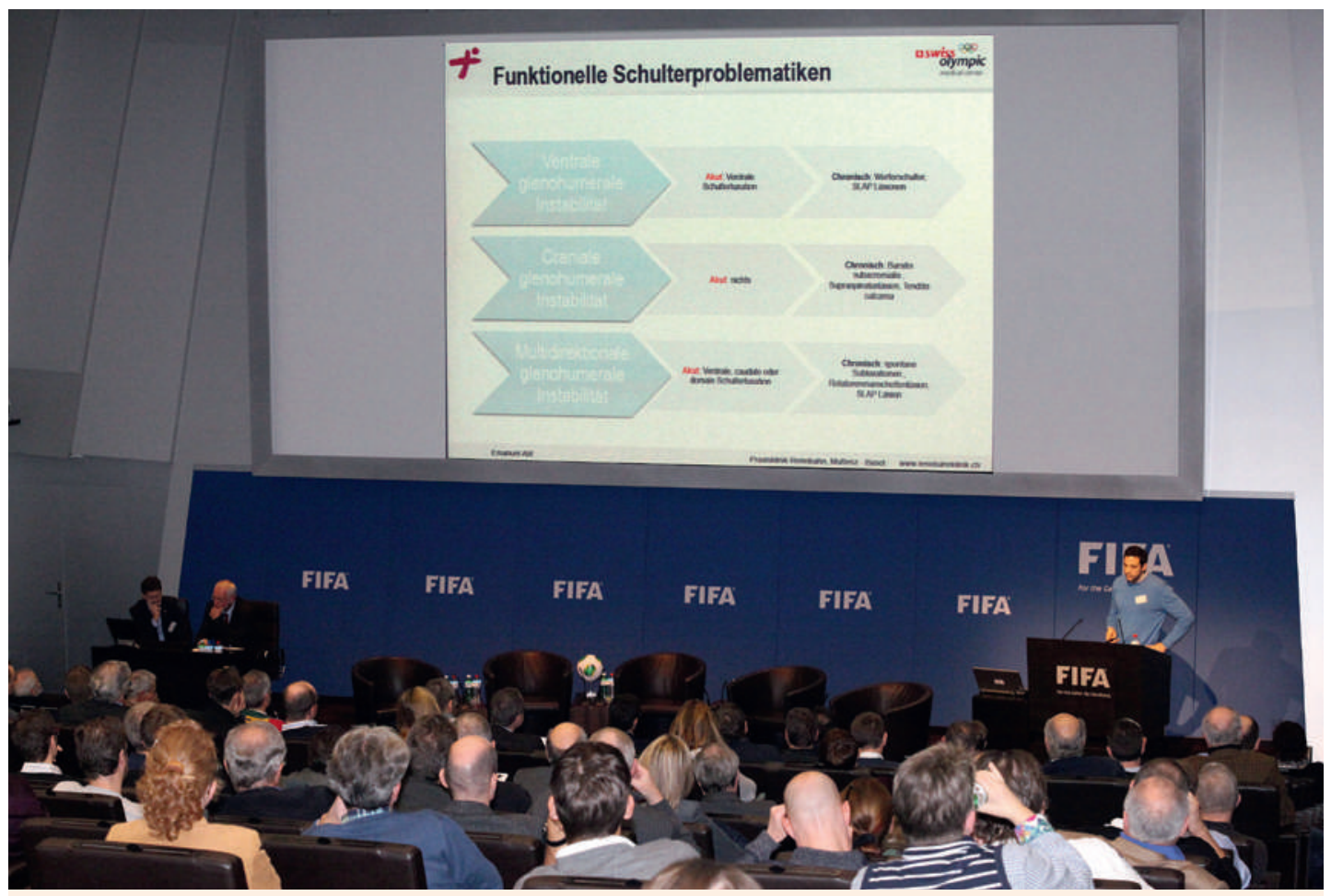

Neben vielen anderen Verletzungen waren auch die häufigen Schulterluxationen ein Thema beim Symposium «Sportverletzungen» in der Zürcher FIFA-Halle. 


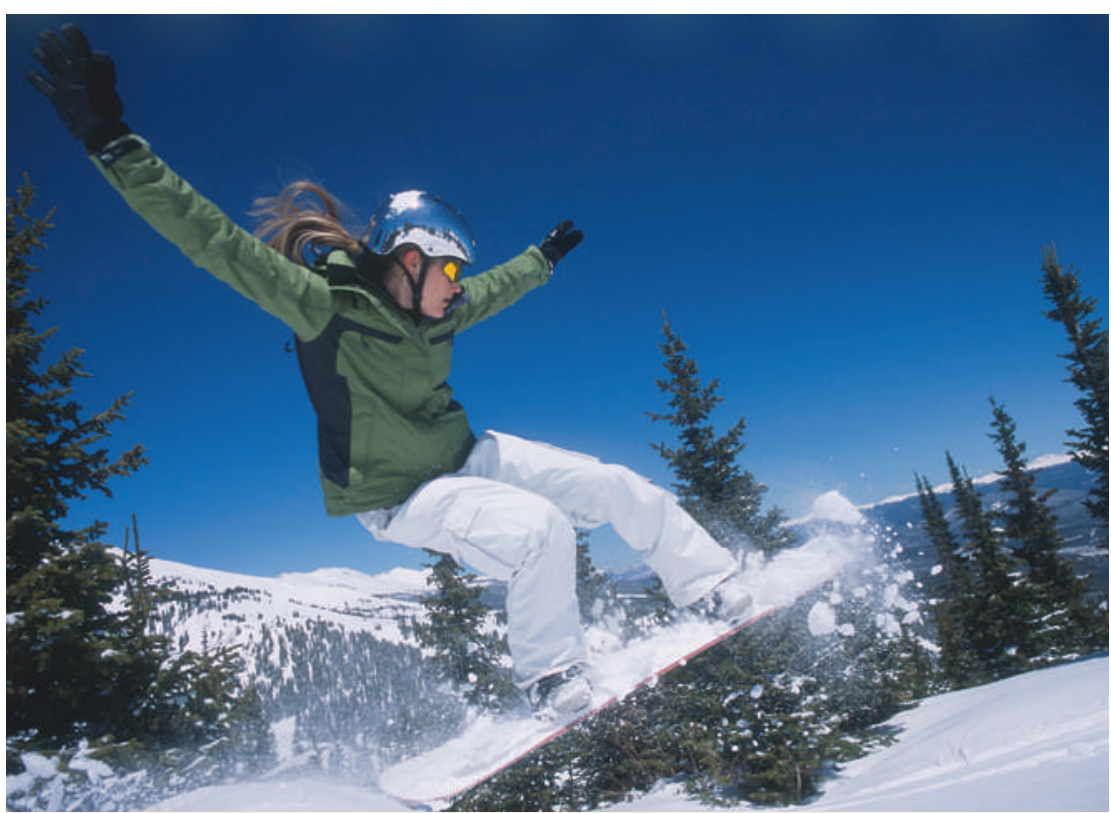

Hoffentlich geht es gut! Auch moderne Rückenprotektoren können axiale Traumen an der Wirbelsäule oder lumbale Frakturen nicht verhindern.

kende Regel eine Sportpause (physical and mental rest) von 7 Tagen und eine symptomangepasste Rückkehr in den Alltag. Immerhin führt eine leichte traumatische Hirnverletzung, LTHV, in einem Prozent zu einer Operation und hat eine Mortalität von $0,1 \%$ (Vos). So ganz banal sind also die «Gehirnerschütterungen» nicht, und auch ein Helm kann sie nicht verhindern, wohl aber die Inzidenz der schwereren Schädelhirntraumen senken. Und so braucht die Rehabilitation der posttraumatischen kognitiven Beeinträchtigung (Iverson) einfach diese 7 Tage, denn «the brain is not a mental muscle» (Ponsford). Und auch unter diesem Aspekt sind body checks im Frauen-Eishockey verboten (Koch). Die Befürchtungen allerdings, dass vor allem rezidivierende LTHVs in einer Demenz münden, entpuppen sich bei kritischer Sichtung als äusserst fragwürdig (McCrory).

Ein Dilemma besteht bei der Behandlung der häufigen Schulterluxation, denn die Reluxationsrate beträgt je nach Alter bis zu 90\%, und auch der ursprüngliche Enthusiasmus für die temporäre Fixation in Aussenrotation ist in der Zwischenzeit wieder abgeflaut, so dass sich heute eine differenzierte, patientenbezogene Abklärung (MRI?) und Behandlung (arthroskopisch oder offen) empfiehlt (Waibl). Revolutionäres hat sich in den letzten Jahren bei der proximalen Humerusfraktur nicht ereignet, die Neer'schen Regeln zur Evaluation einer Operationsindikation sind, wenn auch leicht abgeändert, noch valabel (Acklin), und der physiotherapeutischen Behandlung kommt ein ganz wichtiger Stellenwert zu (Abt). «Die Physiotherapie können Sie getrost den Physiotherapeuten überlassen» hiess es dann später bei den Knieverletzungen (Brühlmann).

Nicht unbedingt der Industrie überlassen sollte man hingegen die Skigestaltung, denn dass schma- lere, weniger taillierte und längere Skis doch einen Einfluss auf die Anfälligkeit von Knieverletzungen haben, weiss man aus den Untersuchungen im Skiprofisport (Spörri). Und damit ist man plötzlich an die alten Hickoryskis erinnert mit den einfachen Flexbindungen und an die Zeit, als noch keine Ratracks die autobahnähnlichen Pisten in der Nacht für den Touristenansturm am nächsten Tag präpariert haben. Machte damals der Wintersport weniger Spass? Wahrscheinlich brach man sich dabei eher das Bein, brauchte dafür aber keine der heute gebräuchlichen Kreuzbandplastiken, die im Langzeitverlauf mit einem 60\%igen Erfolg auch nicht immer die erwarteten Resultate ergeben. Noch weiss man nicht sicher, ob die neue kreuzbanderhaltende Operationstechnik «dynamische intraligamentäre Stabilisierung, DIS» (Eggli) die revolutionäre «Erfindung» unseres Jahrhunderts sein wird, schonender, weniger invasiv ist sie jedenfalls, was ja ganz dem heutigen Trend zur therapeutischen Minimalinvasivität entspricht. Dies empfiehlt sich auch bei den dramatisch zunehmenden, komplexen Tibiaplateau-Frakturen (eine Folge der Tempobolzerei mit den Carvingskis auf den Pisten?), die den Operateur fordern, den Physiotherapeuten und nicht zuletzt den Patienten durch eine oftmals monatelange Rehabilitation (Zappe).

Es ist in diesem Zusammenhang eigentlich erstaunlich (vielleicht aber eben grad nicht), wie schwere Verletzungen, Verletzungen aus adrenalinkickenden Extremsportarten (Basejumping ...) trotz ihrer Schwere mit modernsten Mitteln in den Osteosynthesetechniken und in den postoperativen intensivmedizinischen Massnahmen zu einem erstaunlichen Ergebnis führen können (Keel). Und man gebe sich nicht der Illusion hin, dass die modernen Rückenprotektoren axiale Traumen an der Wirbelsäule verhindern können, die thorakal-lumbalen Frakturen beim Reitsport und die lumbalen bei Skifahrer und Snowboarder (Benneker). Aber auch lebensverändernde Verletzungen mit einer Plegie erlauben heutzutage mit verschiedenen Massnahmen eine Reintegration in einen (veränderten) Alltag (Moulin), was einen zum simpel anmutenden Satz «There is hope» verleiten kann. Aber «wollen» muss dann der betroffene Patient doch noch selbst. Es muss ein (unbändiger) Wille da sein, um selbst in solchen Situationen wieder zum erfüllten Alltag und Sport, sogar Spitzensport, zurückzukehren, denn Angst lähmt (Frei).

Versöhnlich stimmte dieser Schluss des Symposiums, der zeigte, dass mit den heutigen therapeutischen Möglichkeiten auch extremen Verletzungssituationen meist erfolgreich begegnet werden kann. Zur wiederhergestellten Physis (und das braucht vielleicht mehr Zeit, als man sich dies in der Alltagshektik zugestehen will) braucht es aber auch eine entsprechende Psyche, um dem Ziel einer restitutio ad integrum näherzukommen. Die Kombination eben - es braucht einen «mental muscle»! 